\title{
Vestibular Control of Muscular Tone and Posture
}

\author{
Charles H. Markham
}

\begin{abstract}
The vestibulospinal system helps to maintain upright posture and head stability. The semicircular canals and their short latency connections to the neck motoneurons, largely via the medial vestibulospinal tract, respond to angular accelerations so as to stabilize the head in space. The paired otolith organs, the utricles placed approximately horizontally, and the saccules vertically, respond to linear acceleration including gravity. Their influence leads, via the lateral vestibulospinal tract, to excitation of ipsilateral extensor motoneurons of the limbs and trunk, and to inhibition of reciprocal flexor motoneurons. Linear displacement of the otoliths leads to bracing of the limbs and body so as to maintain upright posture, and to extend the limbs so as to help in landing after sudden falls.
\end{abstract}

RÉSUMÉ: Contrôle vestibulaire du tonus musculaire et de la posture. Le système vestibulospinal aide à maintenir la posture en orthostatisme et la stabilité de la tête. Les canaux semicirculaires et leurs connections à latence courte aux motoneurones du cou, qui empruntent en grande partie le tractus vestibulospinalis, répondent à l'accélération angulaire de façon à stabiliser la tête dans l'espace. Les otolithes dont la fonction est jumelée, les utricules placés à peu près horizontalement et les saccules verticalement, répondent à l'accélération linéaire incluant la gravité. Leur influence amène, via le tractus vestibulospinalis latéral, à l'excitation des motoneurones extenseurs ipsilatéraux des membres et du tronc et à l'inhibition des motoneurones fléchisseurs réciproques. Le déplacement linéaire des otolithes amène un raidissement des membres et du corps afin de maintenir la posture en orthostatisme et une extension des membres afin d'aider à l'atterrissage après une chute subite.

Can. J. Neurol. Sci. 1987; 14:493-496

Muscle tone refers to tonic activity in muscles, particularly antigravity muscles, and clinically is detected as resistance to passive movement. It is detectable in normal animals and humans at rest, may be decreased in cerebellar disease, and increased in two forms, rigidity and spasticity. The former consists of constant increase in flexors and extensors as the respective muscles are passively stretched and is seen in nigrostriatal and pallidal hypofunction. Spasticity consists in part of hyperactive stretch reflexes in antigravity muscles and is seen following injury to certain neural pathways which lie in close proximity to corticospinal and corticobulbar pathways.

The vestibular system is a major source of excitatory influence to extensor or antigravity muscles, particularly in axial or proximal limb muscles, and of reciprocal inhibition to certain flexor muscles. Such vestibulospinal activity is best seen when enhanced by transsecting the brainstem rostral to the vestibular nuclei, thus producing decerebrate spasticity. Lesions to Deiters' (lateral) vestibular nucleus or the lateral vestibulospinal tract (LVST) reduce or abolish decerebrate spasticity in the ipsilateral limbs. The LVST activates ipsilateral extensor motoneu- rons directly or via excitatory motoneurons and inhibits ipsilateral flexor motoneurons via inhibitory interneurons. The vestibular complex is a major force in maintenance of upright posture, in antigravity limb responses, and in walking.

Muscle tone refers to tonic activity in muscles and is detected as resistance to passive movement. One component is due to visco-elastic forces in the muscles. In the normal animal or human, greater components stem from the basal ganglia, from pathways in close proximity to the corticospinal tracts, and from the vestibulospinal system.

We are concerned here with the contribution of the vestibular system to muscle tonus, and even more importantly, to posture. The vestibular system responds to changes in linear and angular acceleration and leads to increased tone in extensor, anti-gravity muscles, and reciprocal decreased tone in flexor muscles, so as to maintain upright posture. The vestibular system leads to a short latency increased extensor muscle tone in free fall, apparently in anticipation of landing. In the decerebrate state, sometimes called rigidity but more properly called spasticity, the vestibulospinal system contributes a major ele- 
ment to the increased extensor tone. Let us now look at the several elements of the vestibulospinal system and see how they modulate tonus and posture.

There are two major types of vestibular end organs, the semicircular canals which respond to angular acceleration of the head, and the utricular and saccular otoliths, which are activated by linear acceleration including gravity. The semicircular canals are embedded in the skull in the temporal bones and the three canals on one side of the head are approximately orthogonal to each other, and very approximately parallel to ones on the other side of the head. In ordinary life, their receptor cells respond to very brief and high angular accelerations which lead to increases or decreases in the tonic firing of the first order vestibular nerves, dependent on the direction of rotation. Because of the mechanics of the end organ, the first order canal afferents relay to the brainstem a signal related to head velocity. All four vestibular nuclei receive this information, with those terminating in the medial and lateral (Deiters') nuclei being of particular importance to the neck, trunk and limbs.

The otolith system consists of two receptors on each side, a saccule and a utricle. Each has a layer of calcareous otoconia embedded in a fibrogelatinous matrix, into which the hairs of the receptor cells protrude. The orientation of the receptors determines whether they are depolarized or hyperpolarized as linear acceleration displaces the immediately overlying otoconia. The utricle is shaped like a shallow bowl and is fixed in the skull in an approximately horizontal plane. The saccule has an upward sweep at its forward end and is placed approximately vertically.
Whereas each organ has receptors oriented throughout $360^{\circ}$, the utricle has most of its receptors oriented medially or laterally, and the saccule up or down. The primary afferent nerves fire tonically at about $100 \mathrm{spikes} / \mathrm{sec}$ and this rate is increased or decreased depending on whether the receptors they innervate are depolarized or hyperpolarized. The firing rate can be modified transiently by brief linear accelerations or can be maintained at a new firing rate by constantly applied linear acceleration, for example, gravity. The first order afferents terminate mostly in Deiters' nucleus.

Vestibular information destined for the spinal cord is carried by the lateral vestibulospinal tract (LVST), the medial vestibulospinal tract (MVST) and at least in part by the reticulospinal tract (RST). Much of the following is taken from Wilson and Melvill Jones.'

The LVST originates from cell bodies in Deiters' nucleus and descends entirely ipsilaterally. Its axons travel in the ventral funiculus, quite close to the surface of the cord. Its fibers have a wide range of conduction velocities with the average being quite fast at about 90 meters $/ \mathrm{sec}^{2,3}$ Some axons pass to the sacral cord and some terminate more proximally. Many axons have collaterals leaving the stem axon at right angles ${ }^{4}$ (see Figure 1). Some stem axons have axon collaterals terminating in both the lumbar and cervical cord, clearly indicating that the same vestibulospinal message is carried to both cord levels. Physiological and anatomical evidence indicate some axons terminate directly on limb extensor motoneurons in Rexed's lamina $I X^{4,5}$ while others end on excitatory interneurons which

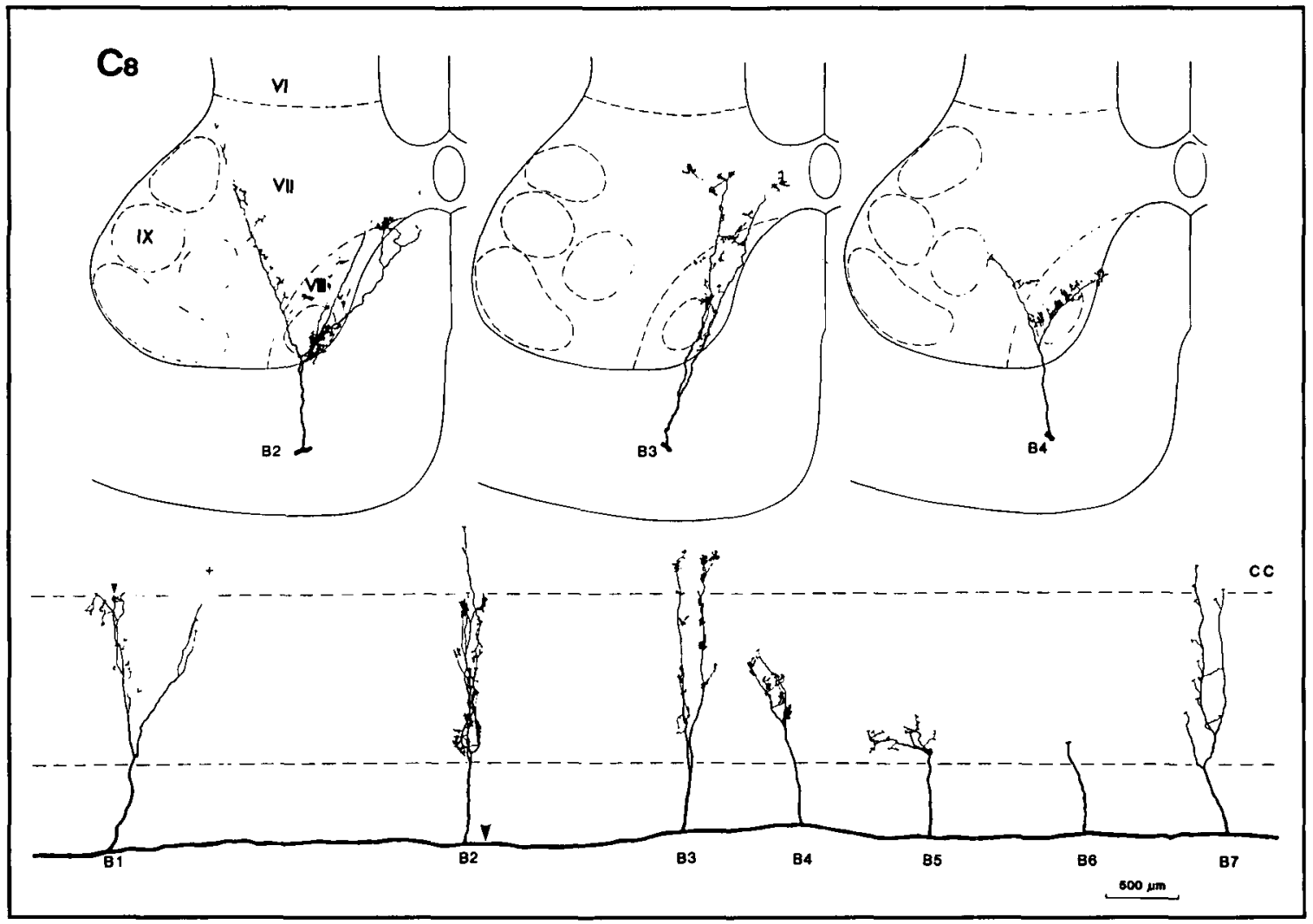

Figure 1 - Reconstructions of axon collaterals from a single LVST axon at C8. Upper drawings are reconstructions in the transverse plane of B2-B4 axon collaterals shown in the sagittal plane in the lower drawing. The lower border of the central canal (CC) and the lower border of the ventral horn are indicated by the dashed lines. The arrow indicates the injection site. The stem axon could be traced further rostrally $(5.9 \mathrm{~mm}$ from $\mathrm{Bl})$ and caudally $(2.7 \mathrm{~mm}$ from $\mathrm{B} 7)$. (From reference 4 , with permission). 
in turn terminate on extensor motoneurons. There also appear to be terminations on large neurons, probably axial muscle motoneurons, in lamina VIII. ${ }^{4}$ Other work suggests termination on inhibitory interneurons innervating ipsilateral flexor motoneurons, as well as on excitatory interneurons reaching contralateral flexor motoneurons. Taken together, there is direct and indirect excitation of ipsilateral limb and axial extensor motoneurons and reciprocal inhibition of ipsilateral flexor motoneurons (see Figure 2). Multiple axon collaterals at different cord levels from single stem axons indicate strong unified postural control.

The MVST originates mostly from neurons in the medial vestibular nucleus but also some from Deiters' and the descending vestibular nuclei. Axons pass down the ipsi- and contralateral medial longitudinal fasciculi (MLF), and pass in the medial part of the ventral funiculus of the spinal cord. Most terminate in the cervical cord and directly or indirectly innervate neck extensor or lateral neck motoneurons. Many neck motoneurons are influenced by all six semicircular canals, with a highly specific excitatory or inhibitory drive from canal pairs (see Figure 3).

The reticulospinal tracts (RST) originate from reticular formation neurons that also receive input, in part from the labyrinths. Some neck motoneurons receive input from both LVST and RST. Some RST axons reach all levels of the cord, some terminating on motoneurons receiving, and others not receiving LVST input. Others terminate on interneurons. RST influence is largely on flexor motoneurons. In the lumbar cord they tend to be reciprocally distributed to extensor motoneurons receiving LVST inputs. ${ }^{6}$ While stimulation of Magoun and Rhine's inhibitory region in the medial medulla inhibits segmental monosynaptic reflexes, it is not clear how the vestibular system influences this effect.

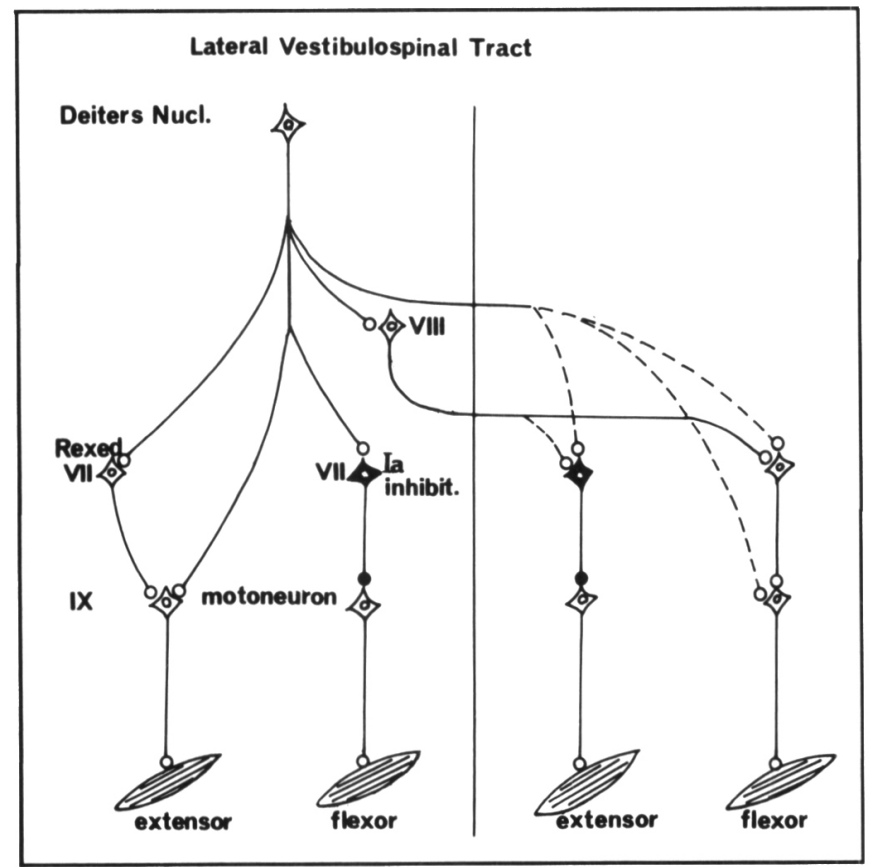

Figure 2 - Diagram of the lateral vestibulospinal track drawn by Tohru Ohgaki (personal communication). Dashed lines are probable connections, solid lines are connections verified either anatomically or physiologically. Roman numerals refer to spinal cord areas after Rexed. Filled cell bodies and terminals are inhibitory, open are excitatory.
Let us now consider some of the postural implications of these pathways. The MVST, as noted above, projects largely to the extensor and lateral neck motoneurons. The cells of origin are mostly second order vestibular neurons receiving input from individual semicircular canals. The synaptic patterns of innervation shown in Figure 3 fit with responses to stimulating individual canals. ${ }^{7}$ The head rotates in the plane of the canal being electrically stimulated. Functionally, natural canal stimulation results in contraction of neck muscles to counter the applied angular acceleration, and thus results in stabilization of the head. This allows a more stable "platform" on which the otoliths, the main source of LVST influence, rest.

The LVST tends to maintain upright posture by tonic excitatory influence, reaching ipsilateral extensor motoneurons and by inhibition, via inhibiting interneurons, of ipsilateral flexor motoneurons. Ipsilateral labyrinthectomy results in sudden loss of this tone (the effects on eyeball stabilization are not considered here). Lesions to Deiters' nucleus or to the LVST produce very similar effects on the trunk and extremities. The body curves to the ipsilateral side and the ipsilateral limbs are flexed. There may be rolling to the ipsilateral side. These effects are more dramatic in rabbits than cats, cats more than man. Rabbits often perform many rolls about their long axes until they become wedged against a wall. There is also rotation of the head if the ipsilateral side of the medial nucleus or whole labyrinth is damaged. After a few days, these effects gradually lessen.

The cellular counterparts of compensation in the vestibulospinal system have been little studied but are probably similar to those seen in the semicircular canal system. In the latter, after ipsilateral labyrinthectomy, Type I vestibular nucleus neurons in mono- or pauci-synaptic connection with the horizontal semicircular canal become silent (no longer fire tonically), and are no

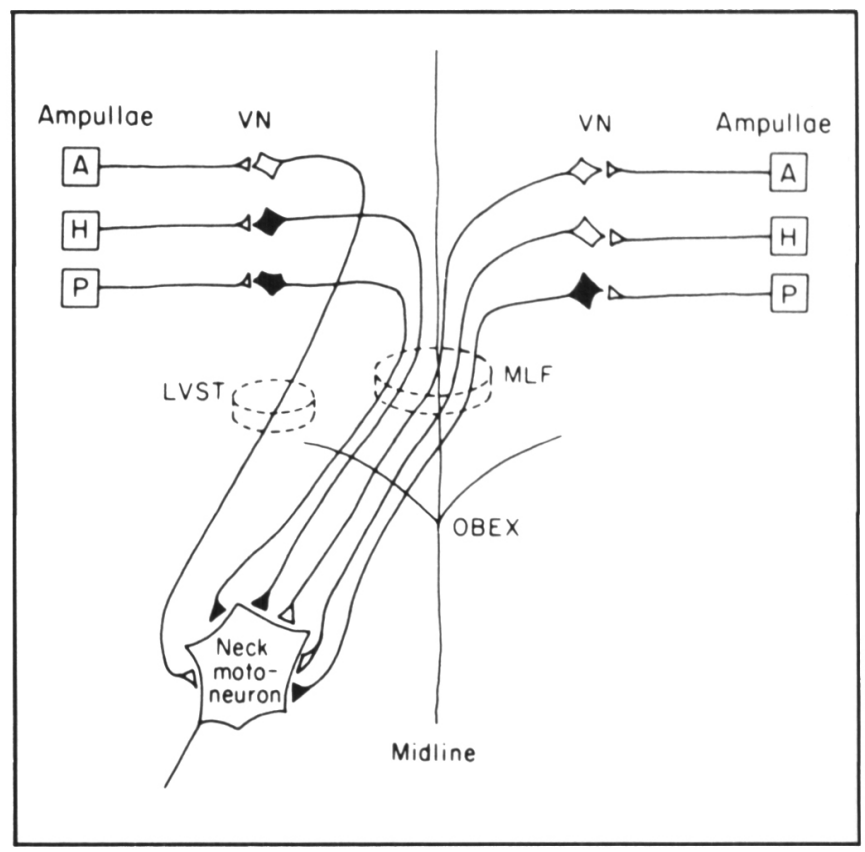

Figure 3 - Schematic drawing of connections between ipsi- and contralateral semicircular canal ampullae and a neck motoneuron. $M L F=$ medial longitudinal fasciculus, $L V S T=$ lateral vestibulospinal tract, $V N=$ vestibular nuclei; $A, H, P=$ anterior, horizontal and posterior canal ampullae. (Reproduced with permission from reference 15). 
longer sensitive to angular acceleration. As days and weeks go by, they regain spontaneous firing and responsitivity to rotation as commissural inhibitory connections from contralateral labyrinth become functionally more powerful. ${ }^{8.9}$

After months go by, recovery becomes nearly complete, such that the cat may lurch to the ipsilateral side only when performing a vigorous maneuver, or the human falls to the ipsilateral side on doing a Romberg test with the feet placed one foot in front of the other.

If vestibulospinal influences are lost because of bilateral labyrinthectomy or streptomycin damage, an individual cannot stand. After some period of time, the person regains the ability to stand, although never with reliability in the dark. Walking becomes possible only in a halting, lurching fashion, with the head bobbing at each change in vertical acceleration as the heels hit the ground.

Another aspect of vestibulospinal function is brought out during vertical stimulation. Fernandez and Goldberg ${ }^{10}$ have shown many first order vestibular afferents respond preferentially to linear accelerations in the vertical direction. From this and from their location in the inferior division in the vestibular nerve, they concluded they were of saccular origin. When cats are dropped from a height, their legs extend and toes fan out. Watt showed EMG's from extensor muscles had a short latency response which was abolished by bilateral labyrinthectomy but not by semicircular canal plugging, the latter blocking canal but not otolith function. " When human subjects were unexpectedly dropped, there was a short latency EMG response from leg extensors, too rapid to come from a segmental myotatic reflex. ${ }^{12.13}$ These otolith-determined responses appear to prepare animal or man for landing from a fall.

The vestibulospinal system has both static and dynamic components. The latter are largely under the influence of the semicircular canals and the former under otolith influence. Money and Scott ${ }^{14}$ demonstrated this in cats placed on a platform which could be tilted. When all the semicircular canals were surgically plugged, thus rendering them inactive, the cats could maintain their balance to slow tilts but would fly off the platform to fast tilts. If the cats then had bilateral labyrinthectomies, thus rendering the otoliths inactive also, the cats would fall from the platform with even slow tilts.

In conclusion, the vestibulospinal system serving the trunk and limbs is under control of the vestibular otolith and canal systems, particularly the former. Both the otolith organs and canals are firmly fixed in the skull. When the otolith system is stimulated by linear acceleration, including gravity, acting on the head, the LVST modifies trunk and limb muscle tonus to maintain posture to respond to sudden falls. Other parts of the vestibular system, the semicircular canals and the MVST, act to stabilize the head of the neck, making a more stable "platform" for the otolith apparatus.

\section{ACKNOWLEDGEMENT}

Acknowledgement is gratefully given to $\mathrm{Dr}$. Tohru Ohgaki for advice and to Mrs. Setsuko Kashitani for secretarial assistance.

\section{REFERENCES}

1. Wilson VJ and Melvill Jones G. In: Mammalian Vestibular Physiology. Plenum Press, New York \& London, 1979: 365.

2. Ito $\mathrm{M}$, Hongo $\mathrm{T}$, Yoshida $\mathrm{M}$, et al. Antidromic and transsynaptic activation of Deiters' neurons induced from the spinal cord. Jap J Physiol 1964; 14: 638-658.

3. Wilson VJ, Kato M, Peterson BW, et al. A single-unit analysis of the organization of Deiters' nucleus. J Neurophysiol 1967; 30: 603-619.

4. Shinoda $Y$, Ohgaki $T$ and Futami T. The morphology of single lateral vestibulospinal tract axons in the lower cervical spinal cord in the cat. J Comp Neurol 1986; 249: 226-247.

5. Grillner S, Hongo T and Lund S. The vestibulospinal tract: Effects on alpha motoneurons in the lumbosacral spinal cord in the cat Exp Brain Res 1970; 10: 94-120.

6. Wilson VJ and Yoshida M. Comparison of effects of stimulation of Deiters' nucleus and medial longitudinal fasciculus on neck, forelimb and hindlimb motoneurons. J Neurophysiol 1969; 32: 743-758.

7. Suzuki J-I and Cohen B. Head, eye, body and limb movements from semicircular canal nerves. Exp Neurol 1964; 10:393-405.

8. Precht W, Shimazu $H$ and Markham CH. A mechanism of central compensation of vestibular function following hemilabyrinthectomy. J Neurophysiol 1966; 29: 996-1010.

9. Markham $\mathrm{CH}$, Yagi T and Curthoys IS. The contribution of the contralateral labyrinth to second order vestibular neuronal activity in the cat. Brain Res 1977; 138: 99-109.

10. Fernandez C and Goldberg JM. Physiology of peripheral neurons innervating otolith organs of the squirrel monkey. I. Response to static tilts and to long-duration centrifugal force. J Neurophysiol 1976; 39: 970-984.

11. Watt DGD. Responses of cats to sudden falls: An otolith originating reflex assisting landing. J Neurophysiol 1976: 39: 257-265.

12. Melvill Jones $G$ and Watt DG. Observations in the control of stepping and hopping movements in man. J Physiol 1971a; 219: 709-727.

13. Melvill Jones $G$ and Watt DG. Muscular control of landing from unexpected falls in man. J Physiol 1971b; 219: 729-737.

14. Money KE and Scott JW. Functions of separate sensory receptors of non-auditory labyrinth of the cat. Am J Physiol 1962; 202: 1211-1220.

15. Wilson VJ and Maeda M. Connections between semicircular canals and neck motoneurons in the cat. J Neurophysiol 1974; 37 : 346-357. 